Martina King, Jesko Reiling

Das Text-Kontext-Problem in der literaturwissenschaftlichen Praxis: Zugänge und Perspektiven

\begin{abstract}
This article begins by using representative examples to present an overview of the diverse ways in which contextualization is practiced in literary studies. Under the rubric of the terms suniversalı (indifferent) and >complementary (distinkt) contextualism, antihermeneutic and hermeneutic approaches are interrogated with respect to their premisses, methods, and intended insights. >Universal contextualism « refers to those post-structuralist methods that operate with a relational concept of the sign and assume that all texts, indeed all the material artefacts of a culture have the same ontological status. A material manifestation of context, or intertextuality, is not assumed here; the aim is insight into regularities of discourse and into media practices. >Complementary contextualism ‘ is intended in its weak form to refer simply to making an indispensable distinction between a text and its contexts - in this case generally in the form of texts - and in its stronger, more clearly hermeneutic version to refer to making a distinction and setting up a hierarchy between a text and its textual or extratextual environment.

The focus of attention is progressively narrowed in the course of the article: the forms and manifestations of universal contextualism are excluded from the remainder of the discussion insofar as the concepts of text and context no longer function as a complementary pair if one ceases to assume a hierarchy, indeed a distinction, between the (literary) text and the connections that explain it. Approaches that go down this path may reveal the practices of discourse in a universe of texts of equal status, but they do not draw on contexts to interpret texts or elucidate the meanings of texts.
\end{abstract}

In the next step, the weak form of complementary contextualism - Stephen Greenblatt's New Historicist approach - is distinguished from stronger versions of complementary contextualism. In abandoning textual autonomy and textual authority and adopting a certain formal-aesthetic indifference, New Historicism dispenses with a strong concept of the text, even if its concentration on canonical authors means that it proceeds in a distinctly literature-centred fashion. Its intended insight, nonetheless, is not the understanding and improved understanding of the literary text in the context in which it originated, but instead > understanding differently $<$ and >making new voices heard $\iota$ : its aim is to reveal the 
social and cultural conditions of possibility of canonical points of textual reference, with the texts and their generally intertextual contexts mutually conditioning one another in the sense of a scirculation of social energies and thus unable to be placed in a hierarchy. Stronger forms of complementary contextualism, on the other hand, work with a form- and language-oriented concept of the text that, depending on whether their interest is defined by authors or problems, can refer respectively to a narrow domain of canonical texts or to an extended domain of high literature and commercial literature. These forms of contextualist literary analysis, though, share premisses of understanding and textual meaning, as well as hermeneutic and philological tools with which reference is made to texts and their extra- and intertextual contexts.

Here, nonetheless, a wide spectrum unfolds. It reaches from what tend to be narrow, author-centric approaches guided by concepts such as intention', influences, sedition', and scommentary contextual analysis whose perspective goes beyond the individual subject and has been broadened to address cultural history. It distances itself from strong authorial intentionalism on the one hand and the associativeness of New Historicism on the other, and is to be understood as a culturalistic extension of social history. Finally, drawing on a hermeneutically negotiated, context-sensitive cultural historiography of this kind, three possible criteria are suggested for selecting contexts of an interand extratextual nature: relevance, representativeness, and usefulness. They serve to limit what is per se an unlimited set of textual environments and have previously been formulated in the literature in a similar manner.

At the end of this increasingly focused research review, which draws on the whole range of contemporary approaches to the problem of text and context, before finally foregrounding a contextualism that is philologically grounded and extended to address cultural history, there follows a transition to the three individual articles in the section we have edited. They are concerned with three different possibilities for dealing with contexts of an inter- and primarily extratextual nature, are all located inside the hermeneutic and philological space we have mapped out, and can to this extent be considered an inexhaustive taxonomy of a contextualism that has been extended to address cultural history: society as a context of literature, knowledge as a context of literature, and finally the textcontext relation as a bipartite combination of problem and solution. Whereas the first two approaches involve classes of context that are defined by content, the final one works with a formal definition of scontext and is to this extent a more broadly conceived one, because almost all political, social, epistemic, and abstract quantities that are external to the text can be formulated as problems. The article, to conclude, is intended to provide an overview that brings order to a 
markedly heterogeneous, diverse research landscape, as well as to stake out a grounded position within this heterogeneous field.

Martina King: Institut für Germanistik, Universität Bern, E-Mail: martina.king@germ.unibe.ch Jesko Reiling: Institut für Germanistik, Universität Bern, E-Mail: jesko.reiling@germ.unibe.ch

\section{Begriffliche Klärungen}

Das Text-Kontext-Problem ist so alt wie die Literaturwissenschaft selbst. Wie der individuelle literarische Text mit dem Überindividuellen der ihn umgebenden Kultur oder Gesellschaft vermittelt ist, wie man seiner Komplexität durch Bestimmung dieser Umgebung Rechnung trägt, das ist »eine der ewigen Debatten der Literaturwissenschaft« (Barner 1997, 4). Eine der ewigen Theoriedebatten und gleichermaßen eines der mächtigsten Implikate aller Praxis: Wer literarische Texte analysieren, interpretieren, besser verstehen, anders verstehen, ihre Struktur oder ihre Wirkungsweise freilegen will, der wird, ungeachtet divergenter Erkenntnisziele, in größerem oder geringerem Ausmaß Entstehungskontexte, also die generische Umgebung des Textes heranziehen. Dabei zeigt sich vielfach jene Unverbundenheit von Theorie und Praxis, die in den Literaturwissenschaften allenthalben beklagt wird - möglicherweise aufgrund eines theoretischen Überangebots und Eklektizismus im Rahmen des cultural turn, wo jeder sich wie im Gemüseladen bedienen oder auch darauf verzichten kann -, jedenfalls zeigt sich dieser Hiatus hier besonders deutlich: Kontextualismus ist als Praxis zwar ubiquitär, aber nicht immer theoretisch gedeckt oder methodisch reflektiert. Man macht es, doch ohne zu begründen, warum und wie man es macht, wozu die selektierten Kontexte dienen und welche interpretatorischen Konsequenzen sich daraus ergeben.

In dieser Ausgangssituation sollen die folgenden Überlegungen zunächst eine gewisse Orientierung bieten - über die gängigen Verfahren im Umgang mit Kontexten, über die implizit oder explizit zugrunde gelegten theoretischen Modelle, ihre Reichweite, Begrenzungen, Probleme, über das jeweilige Erkenntnisinteresse sowie über die Problematik eines praktischen Pluralismus. Daran schließt sich eine Diskussion von drei kurrenten literaturwissenschaftlichen Praxisfeldern bzw. Ansätzen an, die eine rudimentäre Klassifikationsmöglichkeit für Kontexte bieten: >Literatur und Gesellschaft`, >Literatur und Wissen ` und literarische Problemgeschichte. In der Summe erlauben sie die Offenlegung, Ordnung und Einordnung kontextualistischer Verfahrensweisen, wobei sowohl diese Ansätze wie auch unsere eigene argumentative Basis hermeneutisch angelegt sind. Der folgende Überblick und die entsprechenden methodologischen Reflexionen gründen also auf der Dualität von Textbedeutung und Textverstehen. 
Dazu vorab einige terminologische Klärungen. Die Perspektive der folgenden Überlegungen ist doppelt eingeengt - erstens auf literarische Texte, ${ }^{1}$ und zweitens auf ein enges und geschichtsorientiertes Kontextverständnis als einer dem Text äußerlichen Kategorie: Kontext wird mit Lutz Danneberg verstanden als »Menge der für die Erklärung eines Textes relevanten Bezüge« extratextueller oder intertextueller Art (Danneberg 2000, 333). Das weite Kontextverständnis immanenter Ansätze, die intratextuellen und infratextuellen Kontext namhaft machen, bleibt im Folgenden ausgeklammert. Begriffslogisch soll >Kontext als relationales, dreigliedriges Konzept gelten: A ist Kontext von B in Bezug auf C (vgl. ebd.), wobei C als »Verstehensziel« gedacht wird (Lauer 2002, 934). Jeder literarische Text bedarf also - so die hier geltende Prämisse - eines historischen Entstehungskontexts, genauer gesagt einer Vielzahl von Kontexten, deren Ausdehnung je einzeln zu entscheiden und je nach methodischem Hintergrund auch $\mathrm{zu}$ begründen ist. Ansonsten sind seine Lektüren beliebig, aktualisierend, präsentistisch, und je weiter historisch entlegen der Text, desto größer die Verständnisprobleme. Nun lassen sich die explikativen Bezüge, die in ihrer Gesamtheit den Entstehungskontext eines Textes ausmachen, neben den definitorischen Kriterien der Extratextualität oder Intertextualität noch auf andere Weise untergliedern: in Kontexte, die am Autor orientiert sind, am historischen bzw. idealen Leser oder an einem allgemeinen kulturellen Wissen, wobei Letzteres mit Michael Titzmann als »Gesamtmenge der Propositionen, die die Mitglieder der Kultur für wahr halten« gefasst wird (Titzmann 1989, 48).

Damit ist allerdings eine alles andere als selbstverständliche Annahme verbunden: nämlich dass literarische Texte etwas bedeuten, das es zu verstehen gibt und das gegebenenfalls durch Rekonstruktion historischer Semantik, also unterschiedlichster Entstehungskontexte erklärt werden kann. Bekanntlich gründen aber nicht alle Theoriemodelle bzw. Ansätze, die in literaturwissenschaftlichen Handbüchern oder Forschungsberichten unter das Thema >Text-Kontext fallen, auf dieser Annahme (vgl. Köppe/Winko/Jannidis 2007, 349-369; Jahraus 2007, 26f.); spürbar wird das dann in der praktischen Umsetzung. Viele Literaturwissenschaftler rekrutieren zunehmend abgelegene, originelle und epistemologisch höchst voraussetzungsreiche Kontexte, suspendieren aber den Begriff der Textbedeutung. Solchen praktischen Divergenzen zwischen antihermeneutischem und hermeneutischem Zugriff wollen wir uns im Folgenden mit dem deskriptiven Begriffspaar >indifferenter` bzw. >distinkter Kontextualismus` annähern. Unter indifferenten Kontextualismus fallen jene poststrukturalistischen Vorgehenswei-

1 Eine umfassende Sichtung des Text-Kontext-Verhältnisses, einschließlich Musikologie, Altertumswissenschaft, Politikwissenschaft und Linguistik, versuchen Panagl/Wodak 2004. 
sen, die ontologische Statusgleichheit aller Texte, ja aller materialen Artefakte einer Kultur als implizite oder explizite Annahme zugrunde legen; materiale Fixierung des `Kontexts` bzw. Intertextualität zählt hier zu den Voraussetzungen. Mit >distinkter Kontextualismus` soll in einer schwachen Form lediglich die zwingende Unterscheidung von Text und seinen - dann meist textförmigen Kontexten bezeichnet sein, in einer stärkeren, deutlicher hermeneutischen Version die Unterscheidung und Hierarchisierung von Text und seiner textförmigen oder extratextuellen Umgebung. Anhand dieses Begriffspaares ${ }^{2}$ soll kursorisch die Praxis besichtigt werden, wobei wir unsere heuristischen Anschlussüberlegungen auf den Bereich des >distinkten Kontextualismus` beschränken. Wie verfahren also Literaturwissenschaftler im Alltag, jenseits der zum Teil selbstbezüglich geführten Theoriedebatte?

\section{Indifferenter Kontextualismus - Diskursarchäologie}

Auf den ersten Blick scheint der Kontextualismus mit der kultur- und medienwissenschaftlichen Wende im Aufwind: Diskursarchäologie, New Historicism, Kultur- und Wissenspoetiken stellen sämtlich theoretisch fundierte Ansätze dar, die nach der Textzentriertheit von Strukturalismus, Dekonstruktion oder New Criticism entschieden auf breiteste, innovative Kontextualisierung setzen; bevorzugt aus ıinteressanten Bereichen wie Ethnologie, Biologie, Kriminalistik, Ritual- und Religionsforschung, Theatralität, Körpergeschichte, Alltagskultur (vgl. Lauer 2002, 941). Gleichwohl suspendieren besonders Arbeiten, die am Geschichtsbegriff Hayden Whites ${ }^{3}$ und am späten Foucault orientiert sind, nicht selten genau das, was die Text-Kontext-Relation für hermeneutisch orientierte Literatur-

2 Die Überschneidungen mit der von Köppe und Winko vorgeschlagenen zweiachsigen Klassifikation nach den Kriterien ırealhistorisch` (extratextuell) bzw. ssprachlich` (intertextuell) und `Gesellschaft ‘ bzw. `Kultur` (vgl. Köppe/Winko/Jannidis 2007, 337) sind nur partiell: So verstehen wir mit Blick auf den praktischen Alltag Ansätze mit realhistorischem Kontextverständnis ebenso als distinkten Kontextualismus wie neuhistorische Verfahren, die mit intertextuellen Kontexten arbeiten. Unter indifferenten Kontextualismus fallen - unabhängig von Selbst- oder Fremdzuschreibungen mit bestimmten theoretischen labels - lediglich jene dezidiert poststrukturalistischen Zugriffsweisen, die alle kulturellen Artefakte als gleichwertige Intertexte lesen, etwa innerhalb bestimmter Ordnungen oder Genealogien des Wissens, und insofern den Kontextbegriff auch explizit oder implizit ablehnen.

3 Vgl. als locus classicus das Einleitungskapitel »The Poetics of History« von Whites Buch Metahistory (1973). 
wissenschaftler ausmacht - ob Letztere nun unter text- oder problemzentrierter Optik arbeiten: die Hierarchie zwischen dem Text und seinen explikativen Bezügen bzw. die Gliederung in textuellen Vorder- und kontextuellen Hintergrund; ja sogar die Unterscheidung zwischen Kontext und Text als solche. »Wirklichkeit«, schreibt Josef Vogl,

tritt weder in Form von Aussagesätzen noch als Horizont möglicher Erfahrung oder Kontext ins Innere der Texte ein. [...] Literatur ist selbst eine diskursive Formation [...], ist Gegenstand des Wissens [...], ein Funktionselement von Diskursen [...] und wird schließlich durch eine Ordnung des Wissens selbst produziert.

(Vogl 1997, 124)

Bei aller Fülle der von Kulturwissenschaftlern rekonstruierten oder assoziativ aufgerufenen Kontext-Materialien wird demnach ein Universum statusgleicher, notwendig poetischer Texte präsupponiert, die miteinander kommunizieren, ein diskursives Geflecht abgeben, sich zu Genealogien zusammenstellen lassen oder geregelte Praktiken sichtbar machen. Wer etwa das geschichtliche Ereignis des Ersten Weltkriegs als Anthropologie bestimmter Wahrnehmungsfiguren und als Ensemble derjenigen Praktiken konzipieren will, die solche Wahrnehmung ermöglichen, wird beispielsweise die Fotobände von Ernst Jünger, zeitgenössische Schriften zur Akustik sowie Erzählprosa von Kafka und Musil als qualitativ indifferente und diskursiv interdependente Beiträger zu einer »Wahrnehmungsgeschichte der Gefahren « lesen (Encke 2006, 13). Musil-Novellen und Akustik-Schriften verhalten sich hier nicht wie Text und Kontext zueinander, sondern wie gleichrangige Intertexte.

Der Tendenz nach wird in der diskursarchäologischen, kulturpoetischen und mediologischen Praxis also ein distinkter Begriff des Literarischen ersetzt durch ein semiotisches oder medientheoretisches Instrumentarium und philologisches Geschichtsverständnis durch eine latent ahistorische Kulturanthropologie. ${ }^{4}$ Wenn aber »alle kulturellen Zeichen unterschiedslos als Texte« gelten (Lauer 2002, 927), dann kommt mit dem Gegenstand der Literaturwissenschaft auch die

4 Vgl. dazu Gideon Stienings kritische Einlassungen zu einer Fundamentalanthropologie, die eines der konstitutiven Momente einer auf Foucault aufbauenden Kulturwissenschaft sei (Stiening 2009, 41-46). Natürlich gibt es Ausnahmen wie die Décadence-Monographie von Kafitz, die unter diskursarchäologischer Optik ein dezidiert literaturgeschichtliches Phänomen - die französische und deutsche Décadence - quellengesättigt neu sichtet und u. a. auf eine Kontextualisierung kanonischer Texte von Hofmannsthal, Mann und Schnitzler hinausläuft. Es ist gleichwohl vielsagend, dass dieses Verfahren mit Blick auf den diskursarchäologischen Mainstream als »antizyklisch« bezeichnet wurde (Pross 2005, [9]). 
Literaturwissenschaft selbst abhanden, bzw. löst sich in einer »übergreifenden Metawissenschaft unter Aufhebung spezifischer Disziplinarität« auf (Stiening 2009, 38); dann erübrigt sich auch die Frage nach den Modalitäten kontextualistischer Literaturanalyse, da >Text und >Kontext` nicht mehr als komplementäres Begriffspaar funktionieren (vgl. Fohrmann 1997, 215).

So hängt der Umgang mit Kontexten nicht zuletzt am zugrunde gelegten Textbegriff und ergibt sich aus der programmatischen Indifferenz oder Aufmerksamkeit gegenüber etwaigen Literarizitätskriterien wie etwa Gattung, Stil, Fiktionalität, historische Semantik, Textorganisation; ferner aus der Rolle, die man gegebenenfalls Autor und Leser zuweist. Dabei kann das zugrundeliegende Literaturverständnis eng und emphatisch oder weit und deskriptiv sein, das Korpus am Kanon orientiert oder nicht, ${ }^{5}$ relevant ist offensichtlich die Aufmerksamkeit gegenüber den literarischen Kriterien von Textualität und der symbolbildenden Valenz von Literatur. Die unterschiedlichen Kontextgepflogenheiten, die hier als distinkter bzw. indifferenter Kontextualismus firmieren, erscheinen demnach bis zu einem gewissen Grad determiniert: entweder durch ein distinktes, form- und gattungsorientiertes Verständnis von (literarischen) Texten, das an einen entsprechend referentiellen Zeichenbegriff geknüpft ist, oder aber durch Semiotik und einen relationalen Zeichenbegriff. ${ }^{6}$ Fließende Übergänge zwischen den beiden Bereichen sind dabei mitzudenken: So verabschiedet der New Historicism mit der Aufgabe von Textautonomie und Textautorität und einer gewissen formalästhetischen Indifferenz zwar auch den literarischen Textbegriff. ${ }^{7}$ Allerdings wird ein tendenziell enges, emphatisches Literaturverständnis mit der Fokussierung auf kanonische Autoren der englischen Renaissance und schließlich auch anderer Epochen - Shakespeare, Spenser, Dickens, Lukrez (vgl. Greenblatt 1988; Montrose 1986, 1996; Gallagher/Greenblatt 2000, 163-211; Greenblatt 2011) - quasi durch die Hintertür wieder hereingeholt. Zwar sind Text und Kontext

5 Winko, Jannidis und Lauer unterscheiden zwischen emphatischem, engen und deskriptivem, weiten Literaturbegriff. Letzterer impliziert einen erweiterten, nicht mehr am Kanon orientierten Gegenstandsbereich (Winko/Jannidis/Lauer 2006, 146-152).

6 Für Jahraus tritt mit der kulturwissenschaftlichen Erweiterung der Literaturwissenschaft, den daraus resultierenden Neukonzeptualisierungen der Text-Kontext-Relation und den entsprechenden Kontroversen zwischen Philologie und Kulturwissenschaft ein Kernproblem zutage, nämlich »die Frage, was denn überhaupt ein literarischer Text sei« (Jahraus 2007, 36).

7 »The great attraction of this authority is that it appears to bind and fix the energies we prize, to identify and stable a permanent source of literary power, to offer an escape from shared contingency. This project, endlessly repeated, repeatedly fails for one reason: there is no escape from contingency.« (Greenblatt 1988, 3). 
programmatisch enthierarchisiert, insofern Greenblatt eine bloß sakzidentelle Beziehung`des Kontexts zum Text oder die autonomieästhetische Separierung von >Vorder- und Hintergrund als reduktiv verwirft (vgl. Greenblatt 1988, 95). Gleichwohl bleiben beide deutlich getrennt, auch das ist noch als distinkter Kontextualismus zu sehen. Der New Historicism entprivilegiert Literatur und ist dennoch, anders als die diskursarchäologische Forschungspraxis zur Geschichte des Körpers, des Strafens, des ökonomischen Wissens etc., als Programm des Anders-Verstehens explizit literaturzentriert.

\section{Distinkter Kontextualismus - zwischen Einflussforschung und New Historicism}

In den folgenden Abschnitten wird der indifferente Kontextualismus, der jegliche qualitative Differenz in einem unhintergehbar pan-poetischen Universum von Texten und damit sich selbst aufhebt, als Paradoxon aus unseren Überlegungen ausgeklammert bleiben. Zunächst soll ein Überblick gegeben werden über das weite Spektrum dessen, was als distinkter Kontextualismus forschungspraktisch in Erscheinung tritt; denn das einzig verbindende Merkmal ist hier die Trennung von Text und Kontext. Ansonsten ist ein schier unbegrenzter Pluralismus zu verzeichnen. Er reicht von engen, intentionalistischphilologischen Modellen, geschult an Leitbegriffen wie >Autor`, >Einfluss`, `Edition` und ১Kommentar`, bis zum weiten methodischen Programm des New Historicism; und damit von extratextuellen Kontexten bzw. «nicht-textuellen Gegebenheiten« (Danneberg 2000, 334), etwa politischen, sozialen, wissenschaftlichen, kulturellen Ereignissen, Stimmungslagen, Mentalitäten bis zu ausschließlich textförmigen Kontexten bzw. einem assoziativen Intertextualismus, ferner von der Hierarchie zur Gleichrangigkeit. Dazwischen tut sich eine weite Strecke unterschiedlich strenger Plausibilitätsstandards und Selektionskriterien auf: Am einen Ende zielt eine subjektzentrierte Literaturkonzeption auf die personale Fixierung von Kontexten bzw. auf den positiven Nachweis des Autorwissens um Ereignisse, Sachverhalte, kulturelle Bestände. In der Mitte des Spektrums findet sich ein weniger reduktives Forschungsprogramm, das als »kulturwissenschaftlich erweiterte Kontextanalyse« (Vollhardt 2004, 32) einerseits vom strengen Autorintentionalismus, andererseits von neuhistorischer Assoziativität abzugrenzen ist und sich als kulturalistische Fortschreibung der Sozialgeschichte versteht. Unter Einhaltung philologischer Standards zielt eine solche disziplinenbewusste Interdisziplinarität darauf, »möglichst alle erforder- 
lichen Kontexte [zu rekonstruieren] und in ihrer Funktion für die Texte [zu interpretieren]« (Stiening 2009, 8). Anvisiert ist dabei letztlich eine Kulturgeschichtsschreibung, die sich durch die feine aber substantielle Trennlinie des unterschiedlichen Geschichtsbegriffs und der unterschiedlichen Erkenntnisleistung, die man dem Begrifflichen zuschreibt, von den Kulturwissenschaften getrennt sieht.

Als Beispiel für einen solch kulturgeschichtlich erweiterten, interdisziplinären Kontextualismus sei eine Studie zur >Folter in der Literatur` (Kramer 2004) vom 18. Jahrhundert bis zur Gegenwart genannt, da sie mit ihrem Gegenstand weit in die kulturalistischen Bezirke der Körper- und Strafgeschichte hineinreicht. Gleichwohl wird hier keine Geschichte des gefolterten Körpers, sondern eine Geschichte der Darstellungen des gefolterten Körpers, und nochmals differenzierter: der erzählliterarischen Darstellungen des gefolterten Körpers vorgelegt. Diese schon im Untertitel Darstellung in der deutschsprachigen Erzählprosa anklingende Begriffssensibilität ist programmatisch für ein differenzierendes Vorgehen auf verschiedenen Ebenen, das zwischen Geschichte und transhistorischer Kulturanthropologie, realweltlicher und »imaginärer Dimension der Folter« (ebd., 17), Sachen und Zeichen, ferner zwischen verschiedenen Forschungsdisziplinen ${ }^{8}$ und verschiedenen Textsorten unterscheidet; in logischer Konsequenz sind literarischer Text und Kontext hierarchisiert. In kritischer Auseinandersetzung mit Foucault wird dessen Diskursarchäologie im Hinblick auf eine »Verknüpfungsmöglichkeit von Diskurstheorie und kritischer Hermeneutik« (ebd., 21) überschritten, denn kulturwissenschaftlich erweiterter Gegenstandsbereich und formorientiertes Textverständnis, orientiert an »Semantik, Rhetorik, Narratologie und Poetik« (ebd., 22), kommen widerspruchsfrei zusammen. Es resultiert eine >literaturzentrierte Diskursgeschichte`, die trotz kanonischem Quellenkorpus transsubjektiv und autorfern angelegt ist; die allerdings das Problem der letztlichen Unvereinbarkeit von antihermeutischen und hermeneutischen Kontextverfahren sichtbar macht, deren Literatur-, Geschichts- und Zeichenkonzeptionen fundamental auseinanderklaffen. Denn in der praktischen Textanalyse erscheint die Foucault-Orientierung dann weit geringer als in der einleitenden methodologischen Reflexion. Freigelegt werden weniger diskursive Regelmäßigkeiten und mediale Praktiken, denen sich das Reden über Folter verdankt, sondern die Bedeutung, Eigengesetzlichkeit und Sprachgebundenheit literarischer Texte im Lichte ihrer juristischen, politischen,

8 Nämlich zwischen Politologie, Soziologie, Historiographie und Literaturwissenschaft (vgl. Kramer 2004, 15f.). 
philosophischen und alltagskulturellen Entstehungskontexte; dabei ebenso die diskursive Relativität und Relationalität von Literatur. ${ }^{9}$

Eine solch balancierte Mittelposition zwischen kulturgeschichtlicher Breite und philologischer Plausibilität, wie sie hier exemplarisch verwirklicht ist, fällt in etwa mit dem zusammen, was Olaf Krämer jüngst in einem instruktiven Aufsatz zur aktuellen Forschungspraxis als »Korrelations-Ansatz« bezeichnet hat (Krämer 2011, 85) - nur unter etwas anderem Fokus. Gefragt wird nicht nach dem Selektionsmodus von Kontexten, sondern nach der Beziehung zwischen Literatur und der Kontextklasse ‘Wissenschaften`. Um angenommene Ähnlichkeiten bzw. Differenzen zwischen Wissenschaft und Literatur zu beschreiben, würde ein bestimmter Typus literaturwissenschaftlicher Arbeiten "literarische und wissenschaftliche Systemzustände oder Entwicklungsreihen [...] in einen erklärenden Zusammenhang « bringen (ebd., 86), ohne jedoch die Eigengesetzlichkeit und den autonomen Status von Literatur zu verabschieden. Aus der Annahme einer simultanen Koppelung und relativen Autonomie von Literatur und ihrer Umgebung >Wissenschaft` resultiert, dass Beziehungen zwischen beiden mehrfach vermittelt seien, durch literaturimmanente Faktoren - »bereichsspezifische Regeln, Institutionen, Wertmaßstäbe« (ebd., 94) - und durch externe Dynamiken im Wissenschaftssystem; monokausale Einflussthesen werden dabei ebenso vermieden wie die Flucht in ein Universum des Ungeschiedenen. Dehnt man Krämers Deskription dessen, was sich auch als sinnvolles Desiderat bezeichnen ließe, auf andere extratextuelle Kontextklassen von Literatur, vor allem auf `Gesellschaft` aus, resultiert jene hermeneutisch vermittelte Kulturgeschichte, die etwa Vollhardt und Stiening im Blick haben.

Auf dieses Desiderat zielt auch unser Beitrag, wenn am Ende drei Praxisfelder als methodische bzw. proto-methodische Angebote zur strukturierten Kontextualisierung von Literatur vorgestellt werden: neben Krämers Gegenstand >Literatur und

9 Methodisches Prinzip der Studie ist eine epistemologisch funktionale Hierarchisierung von Texten und ihren Kontexten: Erzählprosa von Hoffmann, Tieck, Kafka, Jünger wird auf politik-, rechts- und philosophiehistorische Schriften, ferner auf historische Vorgänge wie die Abschaffung der Folter oder die Errichtung von Konzentrationslagern bezogen, und zwar mit dem Erkenntnisziel, die eigengesetzliche Transformationslogik von Kontexten durch Literatur sichtbar zu machen. Phänomene der poetischen Verschiebung, Überwindung oder Umwertung diskursiver Grenzen, der Reflexion, Deutung und Sinngebung politischer, juridischer und alltagspraktischer Gegebenheiten, sowie umgekehrt, die Indienstnahme kontextueller Elemente Metaphern und Narrative des Folterdiskurses - zur sprachlichen Gestaltung fiktionaler Welten bzw. zu einer eigenständigen Ästhetik des Schreckens werden als spezifische literarische Kompetenzen deutlich. 
Wissen noch `Literatur und Gesellschaft`, schließlich der auf einer anderen kategorialen Ebene angesiedelte Ansatz >Problemgeschichte . Sie erlauben, implizieren letztlich sogar kulturgeschichtliche Erweiterungen des Gegenstandsbereiches, jedoch stets auf philologischer, historisch-rekonstruktiver Grundlage. ${ }^{10}$

Jenseits dieser balancierten Mitte ersetzt am anderen Ende des Spektrums Greenblatts neuhistorisches Konzept von (Literatur-)Geschichte autonome Autorschaft durch ein Modell der Zirkulation - von Meinungen, Praktiken, von sozialer Energie. Dabei wird eine mehr oder minder beliebige, der Möglichkeit nach unbegrenzte, in der Realität meist partikulare Menge von textförmigen, sanekdotischen Kontexten aus Hoch- und Alltagskultur aufgerufen. Erkenntnisziel ist es, die Möglichkeitsbedingungen des Referenztextes freizulegen, wobei sich Text und kontextuelle Umgebung wechselseitig bedingen, folglich auch nicht hierarchisierbar sind. So unterschiedlich also die methodischen Prämissen und Plausibilisierungsstandards, so verschieden auch die hermeneutischen Basisannahmen: Sie variieren zwischen der Zielvorstellung des Angemessenund Besser-Verstehens bei autornahen Kontextrekonstruktionen, deren Selektion auf Repräsentativität angelegt ist, und derjenigen des Anders-Verstehens oder Fremd-Machens im New Historicism; dessen Kontextselektionen zielen auf Marginalität, Okkasionalität und Originalität. Reguliert wird der Verstehensprozess also einerseits durch die hermeneutische Annäherung, die etwas als wahr und richtig Gedachtes vertieft, und andererseits durch eine alternative und dezidiert subjektkritische Sichtweise auf den Text, der sich einem verschütteten Geflecht sozialer, ökonomischer und kultureller Bedingungsfaktoren verdankt.

Probleme finden sich an beiden Polen des Spektrums. Mit dem engen, am Autor orientierten Kontextverständnis wird unter Umständen die Vielfalt an Konnotationen eines Texts verfehlt, die den >kulturellen Paradigmen` der Entstehungszeit bzw. dem überindividuellen kulturellen Wissen, seinen Assoziatio-

10 Es soll in diesem Zusammenhang darauf hingewiesen werden, dass die Debatte um den Modus kontextualistischer Verfahren zwischen Autorintentionalismus und Diskurszentriertheit auch an anderer Stelle formuliert wurde - und zwar von der Cambridge School for the History of Ideas, die die Bedeutungsrekonstruktion politischer Äußerungen notwendig an ihre linguistischen (diskurszentriert) oder ideellen (autorzentriert) Kontexte knüpft. Mit Blick auf beide Versionen, die Diskurszentriertheit Pococks und den Autorintentionalismus Skinners bezweifelt der Ideenhistoriker Bevir zwar den Kontextualismus als Verfahren, das im Sinne einer `logic of discovery Verstehen gesetzmäßig sichert, betrachtet ihn aber als heuristische Maxime: Kontexte sind für Bevir nicht Voraussetzungen des Verstehens, sondern haben explanatorische Funktion (vgl. Bevir 2002, 179). 
nen und Sprachspielen geschuldet ist (vgl. Baßler 2007, 227). Wie man den Risiken des Reduktionismus begegnen kann, zeigt etwa Dieter Martins editionswissenschaftlich informierte Studie Barock um 1800. Sie erhellt die Relevanz des Kontexts `Barockdichtung، für die Literaturproduktion um 1800 konsequent anhand von Rezeptionszeugnissen spätaufklärerischer und romantischer Autoren. Gleichermaßen werden aber die Begrenzungen dieses autornahen Kontextualismus mit Blick auf eine transsubjektive >Funktionsgeschichte des Barocken in der Literatur` überschritten, insofern neben der Quellenforschung die theoretischen Prämissen und das Instrumentarium der Intertextualitätsforschung Anwendung finden (vgl. Martin 2000).

Am anderen Pol des Spektrums birgt das offene Kontextverständnis der Neuhistoriker ohne begrenzende Zulässigkeitskriterien das Risiko der Beliebigkeit. Sein großer Reiz liegt sicherlich in der Praxisnähe Greenblatts und anderer Wissenschaftler, die keine geschlossene Theorie der Kontexterschließung vorlegen (vgl. Baßler 2005, 6-8), sondern pragmatisch den New Historicism als das verstehen, was in jeweiligen Einzelstudien vollzogen wird. Die nicht regelgeleitete, assoziative Aktualisierung jenes Stimmengewirrs, das den Referenztext ursprünglich hervorgebracht und umgeben hat, etwa die Stimmen des Handels, der juristischen Gewalt, der religiösen Praxis, mag zwar die sozialen Energien spürbar machen, mit denen der Text aufgeladen war (vgl. Greenblatt 1988, 1-21; vgl. auch Heitmann 1999). Gleichwohl ist der Preis für ein Textverständnis als >Gewebe ‘ bzw. als »Schnittpunkt vielfältiger, sich überschneidender und wechselseitig beeinflussender (Inter)-Texte und (Inter)-Diskurse« (Neumann/Nünning 2006, 7) unter Umständen jene Willkür, die mit Greenblatts anekdotischem >Dialog mit den Toten ‘ in Verbindung gebracht wird (vgl. Greenblatt 1988, 1-10). Denn es stellt sich doch die Frage, wie jenseits des reinen Freilegens von marginalen Bezügen auch die postulierte Zirkulation sozialer Energien plausibilisiert und nicht nur behauptet werden kann; gemeint sind hier jene reziproken Transferprozesse bzw. >Verhandlungen z zwischen Text und Kontexten, die an die Stelle einseitiger Einflussbeziehungen treten. Mit dem Beharren auf systematischen Wechselwirkungen zwischen Text und Kontexten, die Greenblatts Modell zugrunde liegen, dürfte Intersubjektivität, Konsens- und Anschlussfähigkeit schwer zu erreichen sein. Falls man diese Kriterien überhaupt zu Forschungsdesideraten erklärt - und nicht die epistemische Geschlossenheit des Erratischen, Originellen und Traditionslosen vorzieht - erscheint uns eine philologisch-historische Plausibilisierung abgelegener, innovativer Kontexte, entsprechend der skizzierten Mittelposition, unausweichlich. 


\section{Autor oder Leser, Text oder Symptom, Inhalt oder Form?}

Drei weitere Aspekte der Pluralisierung von distinkter Kontextualität sollen nochmals angesprochen werden - erstens, dass sich die Frage nach dem Kontext am Autor oder auch am Leser orientieren kann. Wer etwa den sozialen, epistemologischen oder kulturellen Kontext realer oder intendierter Leser als Ausgangspunkt wählt, ihre Lektüregewohnheiten, ihren Verstehenshorizont und ihre Erwartungen, der wird sich nicht nur auf die ideen- und semantikgeschichtliche Umgebung des Referenztextes stützen. Der wird darüber hinaus konkrete Rezeptionszeugnisse bemühen, buchmarktgeschichtliche Aspekte hinzuziehen und schließlich ganz andere Erkenntnisinteressen, nämlich wirkungsästhetischer, wirkungsgeschichtlicher oder auch sozialgeschichtlicher Art, mit der Kontextrekonstruktion verbinden als ein hermeneutischer Zugriff, der aus der Interpretation Textbedeutung und Verstehen ableitet.

Zweitens können Erkenntnisinteressen und Fragestellungen kontextualistischer Art textzentriert oder symptomatisch-problemorientiert sein und insofern mit unterschiedlich weiten Literaturbegriffen, Gegenstandsbereichen und Verstehenszielen arbeiten; das Kriterium der Kanonizität kann leitend sein oder nicht. Wer etwa der Funktion von Naturwissenschaften im Werk Thomas Manns oder Gottfried Benns nachgeht, der wird mit der Implikation arbeiten, dass dieser Wissenskontext relevant für die Interpretation vieler oder zumindest etlicher Texte des betreffenden Werks ist und deren Bedeutung mit erschließen kann; ja dass die Bedeutung nur dann erkennbar wird, wenn man den historischen Stand der Wissenschaften und das konkrete Autorwissen rekonstruiert (vgl. Herwig 2004; Hahn 2011). Die Beschränkung auf jeweils hoch kanonische Euvres und ihre Verfasser impliziert dabei einen relativ engen, emphatischen Literaturbegriff, eine autorzentrierte Selektion der Kontextbeispiele und ein definiertes Verstehensziel C: das Werkverständnis. Schließlich würde man nicht die gleiche kontextualistische Fragestellung an die umfangreichen Romane Conrad Albertis herantragen; obwohl der Verfasser sicherlich ebenfalls den Werkbegriff für sein Schaffen beanspruchte und der Kontext >Naturwissenschaften`, genauer gesagt Darwinismus, für das Verstehen dieses Werks ebenfalls hoch relevant ist. Vielmehr bietet es sich an, solche nicht kanonisierten Texte unter übergreifenden, symptomatischen Frageperspektiven zu vergleichbaren Textgruppen zusammenfassen und dann etwa nach Interdependenzen zwischen ihnen und dem Kontext 
zu fahnden, etwa der »dichten Interaktion« von Darwinismus und deutschem Naturalismus (Stöckmann 2009, 42). ${ }^{11}$

Ein symptomorientierter Kontextualismus, wiederum in der Nähe von Krämers Korrelationsmodell, impliziert demnach in der Praxis breitere Textkorpora als autor- oder werkzentrierte Fragestellungen und eine Kontextselektion entlang des Kriteriums >kulturelles Wissen`. Schließlich ist historische Repräsentativität der Anspruch auf beiden Seiten, der des Textes und der des Kontexts. Wer beispielsweise verstehen will (C), inwiefern fiktionale Texte den Wandel der Subjektkonzeption im psychiatrisch-anthropologischen Wissenssystem des 19. Jahrhunderts reflektieren, antizipieren, befördern, konterkarieren oder eigenlogisch transformieren, der wird zur Plausibilisierung der angenommenen TextKontext-Beziehungen neben kanonisierten Erzähltexten auch solche unterhalb der Kanonschwelle heranziehen: Raabe, Schnitzler, Fontane und daneben Wilhem Bölsche sowie Hermann Stehr (vgl. Thomé 1993). Die Selektion von Kontextbeispielen aus der Psychiatriegeschichte wird sich nicht oder nicht nur an den Wissensbeständen dieser Autoren orientieren, sondern an »der offiziellen Universitätslehre, [am] diskreten Praktikerwissen [...] und [an] volkstümlichen Vorstellungen« (Thomé 1993, 16f.), die sämtlich in das kulturelle Wissen der Epoche eingegangen sind. Entscheidend ist gleichwohl, dass enger oder erweiterter Gegenstandsbereich und textzentrierter oder symptomorientierter Kontextualismus nicht notwendig mit engen oder lockeren philologischen Standards der Kontexterschließung korrelieren. Sowohl die erwähnten Schriften zu Thomas Mann oder Gottfried Benn wie auch diejenige zur psychiatrischen Anthropologie im Roman des 19. Jahrhunderts können durchaus den gleichen strengen philologischen Standards der Kontextwahl und -limitation verpflichtet sein und ein ähnlich distinktes, formorientiertes Verständnis des literarischen Texts teilen,

11 Ziel der Studie ist eine Neubewertung des Naturalismus als Initiator der literarischen Moderne im Lichte wissens- und philosophiegeschichtlicher Kontexte. In den methodischen Reflexionen des Verfassers zeigt sich die Nähe zu Krämers Korrelationsmodell und eine Abgrenzung von wissenspoetologischen Verfahren: "Dabei handelt es sich ausdrücklich um eine Darstellung von problemgeschichtlichen Grundzügen [...] zunächst, weil die wissenschafts- und philosophiegeschichtlichen Fakten [...] in der literaturgeschichtlichen Konstellation mit dem Naturalismus eine eigene Signifikanz entwickeln. Sodann, weil die spezifischen Homologien zwischen Literatur und kulturellem Wissen an den entsprechenden Stellen im Verlauf der Argumentation zur Sprache kommen; drittens schließlich, weil die Arbeit kein im strengen Sinn >wissenspoetologisches Interesse verfolgt, das die Analyse auch auf das im allgemeinsten Sinn `kulturelle Wissen der Zeit`zu konzentrieren hätte« (Stöckmann 2009, 7). 
nicht aber den zugrunde liegenden Gegenstandsbereich. Hingegen teilen die Beispiele zu Thomas Mann und Gottfried Benn mit der neuhistorischen Analyse von Shakespeare-Dramen, Lukrez' Lehrgedicht De Rerum Naturarum, Dickens' Roman Great Expectations oder auch Edmund Spensers Texten (vgl. Greenblatt 2011, 1988; Gallagher/Greenblatt 2000; Montrose 1988, 1996) die Orientierung an monumentalen Autoren und insofern den gleichen engen, kanonischen Gegenstandsbereich.

Sie divergieren aber denkbar weit in punkto der philologischen Standards und der Selektionskriterien für Kontexte; den Kriterien der Repräsentativität und Relevanz stehen solche der Originalität und Abgelegenheit gegenüber. Fundamental verschieden ist vor allem das zugrunde gelegte Verständnis vom literarischem Text und von literarischer Kommunikation: Thomas Manns Romane und Gottfried Benns Dichtung erscheinen jeweils als geschlossene, selbstreferentielle Gebilde, die naturwissenschaftliche Kontexte nach je eigener symbolischer Logik anverwandeln und transformieren. Mit Shakespeares Dramen, Lukrez' Lehrgedicht, Dickens' Prosa oder auch Edmund Spensers Werk hingegen kommt der literarische Text als eine der vielen möglichen Repräsentationsformen von Kultur im Allgemeinen oder von spezifischen Kulturphänomenen wie Kolonialismus, Sexualität, Ritualität, Religionskonflikten etc., in den Blick. Er ist ohne autonomen Sonderstatus in kulturelle Austauschprozesse eingebunden; etwa zwischen elisabethanischem Theater und Exorzismus-Praktiken oder umgekehrt zwischen antikem Lehrgedicht und den verschiedensten Ausprägungen eines humanistischen Weltverständnisses in der Renaissance, für das der kanonische literarische Text dann zum Kontext wird. ${ }^{12}$

In unseren weiteren Überlegungen wird nun auch dieser schwache Textbegriff der New Historicists ausgeklammert sein - und mit ihm die schwache Form des distinkten Kontextualismus, die auf die Hierarchisierung von Text und intertextuellen Kontexten verzichtet. Der Fokus liegt auf einem distinkten, sprachorientierten Verständnis des literarischen Texts, sei der literarische Gegenstandsbereich eng und kanonbezogen oder erweitert-deskriptiv. Denn für beide Versionen bleibt noch, das ist der dritte Punkt dieses Abschnitts, eine zentrale Frage zu klären: Wie kann die Analyse von Text-Kontext-Beziehungen genau diese Sprachgebundenheit bzw. Sprachverfremdung mitberücksichtigen, die sich als eines der konsensfähigen Kriterien für jeglichen distinkten Literaturbegriff,

12 Laut Fohrmann werde in Greenblatts Shakespearean Negotiations »ein Kunstbegriff [...] nicht mehr oder nur in sehr schwacher Form noch vorausgesetzt« (Fohrmann 1997, 210). 
emphatisch oder deskriptiv, etabliert hat ${ }^{13}$ Für manche Texte scheint die Relation von ästhetischer Form und Kontext unschwer zu plausibilisieren. Wenn man etwa zur Analyse von August Stramms Wortkunstgedichten den politisch-geschichtlichen Entstehungskontext `Stellungskrieg` und den philosophischen Entstehungskontext >Sprachkrise` hinzuzieht, so lässt sich die spezifische Form dieser Texte - schnelle, rhythmisierte Ein-Wort-Kaskaden oder Laut-Reihen - bis zu einem gewissen Grad durch diese Kontexte erklären: Die prononcierte Lautlichkeit und Asemantik, der Grammatikverlust und das ıStaccato ‘ der Texte sind einerseits auf die Sinneswahrnehmungen im Schützengraben, andererseits auf die sprachkritische Kunsttheorie im Sturm-Kreis zu beziehen. Zudem lässt sich diese Verknüpfung von Form und Kontexten philologisch stützen: durch die Briefe, die Stramm 1915 aus den Schützengräben der Westfront und später von der polnischen Ostfront an Herwarth und Nell Walden geschrieben hat (vgl. Stramm 1997, 172-199). Doch abgesehen von solchen Einzelfällen dürfte für Formfragen, solche des Einzeltextes oder auch der Gattung, nicht selten ein methodischer Hiatus verbleiben: zwischen umfänglichen Rekonstruktionen der Textumgebung einerseits, die zum Verstehen des Text-Gehalts, seiner Sinn- und Denkfiguren beitragen, und der semantischen, rhetorischen, kompositorischen Spezifität der Darstellung andererseits. Als bedeutungskonstitutiv lässt sich Letztere von der Textaussage nicht lösen und ist dennoch von der kontextualistischen Perspektive nicht selbstverständlich gedeckt.

Um solchem Reduktionismus zu entgehen, fordern Neumann und Nünning ein hybrides Vorgehen aus kulturalistischer Weite und distinktem, formorientierten Literaturbegriff. Eine kontextualistische Literaturwissenschaft habe einerseits »das Augenmerk konsequent auf die formal-ästhetischen Strategien des Symbolsystems Literatur zu richten «, andererseits »nicht nur von einem weiten Literaturbegriff auszugehen, sondern Literatur auch konsequent als Teil der Medienkultur zu modellieren« (Neumann/Nünning 2006, 12). In diese Richtung einer systema-

13 Zwar ist das Merkmal der besonderen sprachlichen Gestaltung weder hinreichend noch notwendig, da nicht alle Texte, die zur Literatur gerechnet werden, darüber verfügen, z.B. Schemaliteratur, hingegen etliche Texttypen, die nicht zur Literatur gerechnet werden, schon, z. B. Werbungstexte (vgl. Winko/Jannidis/Lauer 2006, 11f.). Unter pragmatischer Perspektive dürfte das Kriterium für die Mehrzahl der Literaturwissenschaftler allerdings leitend sein bzw. Literatur als Prototyp festlegen, so dass man damit arbeiten kann, aber nicht alle Einzelphänomene darunter fallen müssen (vgl. ebd., 12f.). Vgl. auch den Beitrag von Dirk Oschmann in einem aktuellen Band zum Begriff der Literatur, der das Kriterium `Sprachlichkeit` in kritischer Abgrenzung vom kulturwissenschaftlichen Relativismus bzw. vom `practical turn zur Grundlage einer essentiellen Literatur- und Sprachkonzeption macht: Literatur gebe, so Oschmann, »im Modus des Zeigens die Sprache zu erkennen« (Oschmann 2010, 411). 
tischen Vermittlung von Kontexten und ästhetischer Form gehen Überlegungen in der postklassischen Narratologie. Die Konzeption einer 'Semantisierung literarischer Formen` (vgl. Nünning 2000, 361; Erll/Roggendorf 2002, 82f.) zielt u. a. auf eine kulturgeschichtliche Kontextualisierung von Analysekategorien der klassischen Narratologie wie Plot, Perspektive, Fokalisierung, Stimme. Sie dienen dann nicht mehr nur der ahistorischen Beschreibung von Textorganisation bzw. -struktur, sondern der Interpretation des Textes und enthüllen ihre Relevanz für dessen Bedeutungsebene im Licht seiner historischen Umgebung; und unter >Text` fällt ja mit der transgenerischen und kognitiven Erweiterung der Erzählforschung mittlerweile eine Vielzahl von Gattungen, vom Drama bis zur graphic novel. Allerdings handelt es sich bei der kulturgeschichtlichen Narratologie um eine "präzisionsbedürftige Forschungsrichtung" (Erll/Roggendorf 2002, 79), die bislang vor allem empirisch in Einzelstudien realisiert ist.

So macht etwa eine neuere Monographie zur »Krise der Teleologie im literarischen Realismus« (Ajouri 2007) einen extratextuellen, ideengeschichtlichen Kontext von Literatur, den Funktionsverlust des teleologischen Weltbildes im 19. Jahrhundert, einerseits an zahlreichen Dokumenten aus Naturwissenschaft, Philosophie und Ästhetik namhaft und rekonstruiert damit die materiale Basis dieses Kontexts. Andererseits ergeben sich daraus, so der Argumentationsgang, Formprobleme bzw. formale Innovationsimpulse für die Erzählliteratur der Epoche: Narrative werden, entgegen normativer Prämissen des programmatischen Realismus, nicht mehr nur final, kohärent und kontinuierlich konzipiert. Vielmehr zeichnet sich im Licht der ideengeschichtlichen Textumgebung - Feuerbach, Büchner, Darwin, und die Debatte um eine kontingente Natur - das offene, kompilative bzw. montagehafte Erzählen der Moderne ab. So vermitteln etwa anachrone Zeitordnung, dominante interne Fokalisierung und der Verzicht auf Auktorialität in einem Roman Vischers jene »Sprunghaftigkeit und Zufälligkeit» von Ereignissen (Ajouri 2007, 200), die sich aus dem Kontext der Darwinschen Selektionstheorie und dem epistemologischen Primat des Zufalls ergeben. Konsequente Semantisierung narratologischer Befunde im Licht des ideengeschichtlichen Kontexts: Hier tut sich offensichtlich eine fruchtbare Möglichkeit auf, extratextuelle Umgebung und ästhetische Textgestalt aufeinander zu beziehen, und man sieht, dass strukturelle Korrelationen nicht notwendig auf konkrete Intertexte angewiesen sind. Sie lassen sich auch zwischen Texten und den sie umgebenden mentalen Beständen beschreiben. Allerdings spielen philologische Standards gerade dann eine bedeutende Rolle, wenn der weite Transfer zwischen ideen- oder wissensgeschichtlichen Denkfiguren und binnenliterarischer Form im Einzelfall plausibel werden soll. 


\section{Praktischer Pluralismus - methodische Evidenz des Einzelfalls}

Zusammenfassend scheint in der skizzierten Situation des kontextualistischen Pluralismus jeglicher Konsens, ja gar die methodisch-theoretische Begründung eines verbindlichen Verfahrens weder möglich noch sinnvoll, schließlich vermag »ein verallgemeinerndes Text-Kontext-Modell [...] der Vielschichtigkeit des Gegenstandes nicht gerecht zu werden« (Neumann/Nünning 2006, 19). Konsensfähig ist offensichtlich nur die Abwesenheit von Konsens: Auch wenn man laut Baßler vermuten könne, »dass jeder einzelne Wissenschaftler durchaus über ssein` mehr oder weniger explizites Text-Kontext-Modell verfügt«, scheint »ein wie auch immer gearteter methodologischer Konsens [...] nicht zu bestehen" (Baßler 2005, 2). Aus den bisher gemachten Ausführungen geht hervor, dass dieser Pluralismus in der theoretischen bzw. methodischen Heterogenität, vor allem im Dissens zwischen antihermeneutischen und hermeneutischen Hintergrundannahmen wurzelt, die die gegenwärtige literaturwissenschaftliche Praxis prägen - mögen die jeweiligen methodischen Orientierungen nun in den Einzelstudien mitreflektiert sein oder nicht. Vor allem dürfte er ganz einfach darin gründen, dass Kontextbildung zu den traditionsreichsten und selbstverständlichsten Praktiken in den Literaturwissenschaften gehört und dazu tendiert, sich - in unterschiedlichsten Gestalten - in jedwedes methodisch-theoretische >Lager jenseits der reinen Textimmanenz einzuschleichen.

Um trotzdem ein gewisses Maß an wissenschaftlichem Mehrwert zu sichern und nicht in beliebige Idiosynkrasien zu münden, bietet sich ein pragmatischer Umgang mit dem Text-Kontext-Problem an. Wenn Kontextualismus lediglich phänomenologisch als das in den Blick kommt, was - häufig unmarkiert - in der literatur- und kulturgeschichtlichen Alltagspraxis betrieben wird, so lässt sich immerhin das Desiderat einer terminologischen Klärung und Offenlegung des Einzelfalls ableiten. Ziel einer kontextbewussten Literaturwissenschaft sei es, so Neumann und Nünning, »die notorisch vagen Kategorien des New Historicism terminologisch und konzeptuell zu präzisieren « (Neumann/Nünning 2006, 15). Allerdings dürfte sich dieses Ziel eher in der je individuellen methodischen Reflexion, Begründung und Explikation als auf der Ebene methodischer Standards realisieren lassen.

So begründet etwa eine Studie, die der "Literarisierung von Elektrizität und Elektrizitätslehre« (Specht 2010) um 1800 gewidmet ist, erstens die Wahl des literarischen Quellenkorpus; und zwar mit dem personenbezogenen Kriterium der naturwissenschaftlich-poetischen Doppelkompetenz. Es erleichtert die kontextualistische Einbettung "naturwissenschaftlicher Positionen« der Referenzautoren in den allgemeinen Wissenskontext `Elektrophysikı (Specht 2010, 4) und schlägt die 
Brücke zu ihren literarischen Stellungnahmen. Zweitens nimmt die Studie zur historischen Spezifität ihrer Text-Kontext-Relation explikativ Stellung. Hingewiesen wird auf den noch unabgeschlossenen Differenzierungsprozess zwischen Naturwissenschaft, Naturkunde, Naturphilosophie und Kunst, und insofern auf die geschichtlich fixierte Unterscheidungsproblematik von Texten und Kontexten einerseits, von einander verwandten Wissenskontexten andererseits (vgl. ebd., 5-8). Drittens wird in dieser komplexen Gemengelage ein methodischer Rahmen für die Kontextrekonstruktion angegeben: Titzmanns pragmatischer Begriff des kulturellen Wissens, bzw. >dessen, was die Mitglieder einer Kultur für wahr halten` (oder diejenigen einer kulturellen Subgruppe). Daraus geht dann wiederum die Priorität qualitativer vor quantitativer Repräsentanz hervor (vgl. ebd., 20) und insofern ein Such- und Begrenzungskriterium für schriftliche Kontextbeispiele: Die Rekonstruktion des voraussetzbaren elektrophysikalischen Wissens setzt »an Handbüchern und Lexika aus der Zeit an und [stößt] von da aus zu den modellhaften Texten vor[...]« (ebd.). Eine solche gattungssensible Begründung für die Kontextselektion schafft Evidenz, plausibilisiert, klärt auf; nicht zuletzt über die vielschichtige argumentative Rolle des Kontexts. Die wissenschaftsgeschichtliche Umgebung der Texte dient hier nicht nur dem Verstehen und Besserverstehen, sondern erhellt die Funktion literarischer Kommunikation in Bezug auf übergeordnete Strukturen wie Gesellschaft oder Kultur, in welche sie modifizierend eingreift. So wird eingangs die erkenntnisleitende Frage gestellt, inwiefern das Medium Literatur dazu beitrage, »den Ergebnissen und Methoden der Wissenschaft eine allgemeine weltanschauliche Bedeutung beizulegen« (ebd., 3), und in drei umfangreichen, textanalytischen Kapiteln zu Ritter, Novalis und Kleist beantwortet.

Das Beispiel entspricht erneut der oben skizzierten Mittelposition zwischen autornahen und neuhistorischen Verfahren, also dem Korrelationsmodell Krämers. Darüber hinaus zeigt es, dass die hier angesprochene smethodische Evidenz des Einzelfalls`, besonders im Verbund mit philologischen Standards der Kontexterschließung, längerfristige Möglichkeiten für Anschlussforschung bietet: in prospektiver Hinsicht, insofern offengelegte Selektions- und Begrenzungskriterien zu epistemologischen Bezugnahmen einladen; in retrospektiver Hinsicht, insofern innovative neue Kontext-Bezüge in einen akkumulierten Bestand von Forschungserkenntnissen einrücken, der nur aus quellenbewusster, historisch-semantischer Rekonstruktionsarbeit hervorgehen kann. Das derart konstituierte epistemische Kontinuum, das Bausteine zur oben erwähnten, hermeneutisch vermittelten Kulturgeschichte liefert, unterscheidet sich vom programmatischen Partikularismus poststrukturalistischer Zugriffsweisen. Denn radikale Diskontinuität wird von Vertretern der Diskursarchäologie nicht nur in die Geschichte projiziert, sondern auch der eigenen Forschungslandschaft als Signatur aufgeprägt. Es ist eine Diskontinuität des absoluten Individualismus, des Interessanten und nicht selten Opaken, die 
an die Stelle des philologisch-historischen Zusammenhangs tritt und »von Forschungstraditionen entlastet« (Lauer 2002, 935).

Hermeneutische Verfahren hingegen, für die das Primat der Anschlussfähigkeit und des Traditionsbezuges gilt, sind mit einem mehr oder weniger fixen >Bausatz`methodischer Probleme konfrontiert, die je im Einzelfall mit größtmöglicher Evidenz zu klären sind. Gemeint ist damit vor allem der regelgeleitete Einschluss und Ausschluss von Kontexten, genauer gesagt die begründete Limitation eines im Hinblick auf Reichweite und Themenvielfalt unbegrenzten Raumes, der den Text umgibt. Denn zunächst ist zu klären, welches Vorverständnis überhaupt die Ermittlung von Kontexten regeln kann, mittels derer dann die Texte erklärt werden sollen (vgl. Danneberg 2000, 335) - oder auch: Wer noch nichts verstanden hat, kann auch die Kontexte nicht rekrutieren, ohne deren Kenntnis der Text nicht verstanden werden kann. Daher scheint ein heuristisches Rahmenwerk sinnvoll, das die Suche initiiert, organisiert und bis $\mathrm{zu}$ einem gewissen Grad reglementiert, selbst wenn man sich damit in die Bereiche methodischer Normativität begibt. ${ }^{14}$ In diesem Zusammenhang schließen wir Überlegungen zu einigen groben Kriterien an - neben dem bereits diskutierten Postulat der methodischen Evidenz des Einzelfalls.

\section{Kriterien der hermeneutischen Kontext- Erschließung}

In der Praxis muss sich die Rekonstruktion eines extratextuellen Kontexts oder besser, einer nicht materialen Text-Umgebung, etwa einer ideellen, epistemischen oder gesellschaftlichen Konstellation, aus ökonomischen Gründen auf materiale Beispiele für diese Konstellation beschränken. Als mögliche Kriterien für die Selektion und Limitation von Kontexten wollen wir >Repräsentativität‘, >Relevanz` und `Fruchtbarkeit`vorschlagen; natürlich ist diese Liste nicht erschöpfend, kann in unseren Augen allerdings praktisch ein breites Feld der TextKontext-Relationierung abdecken und ist in ähnlicher Weise in der Forschung bereits formuliert worden: Ralf Klausnitzer zufolge sollen Text-Kontext-Verknüp-

14 Und dies gilt ebenso für kulturwissenschaftliche Erweiterungen im Forschungsfeld der Vormoderne wie für die Makroperiode Moderne, der die bisherigen Beispiele entstammen: Die Angemessenheit von Kontextualisierungen könne, so Jan Dirk Müller in einem Band zu einer »kulturwissenschaftlich angeleiteten Mediävistik«, nicht vorweg theoretisch, sondern nur forschungspraktisch entschieden werden und bemesse sich je an der »methodischen Stringenz«, mit der der Forschungsertrag gewonnen worden sei (Müller 2007, VII). 
fungen ökonomisch verfahren, adäquat sein, Text und Kontext in systematische Verweisungszusammenhänge bringen und schließlich Textstellen vollständig erklären (Klausnitzer 2012, 73). Besonders die Kriterien der Ökonomie und der Adäquatheit scheinen den von uns vorgeschlagenen der Repräsentativität und der Relevanz vergleichbar zu sein. Dabei bezeichnet >Repräsentativität` eine normative Relation zwischen Kontextbeispiel und Gesamtkontext, >Relevanz` eine normative Beziehung zwischen Kontextbeispiel und literarischem Text und Fruchtbarkeit den erwarteten Erkenntniszuwachs aus einer relevanten Text-Kontext-Relation. Repräsentativität kann nun quantitativ verstanden werden, als Querschnitt durch eine angenommene große Zahl kontextueller Belege, etwa wenn es um Äußerungen in der Massenpresse des Fin de Siècle zur Frauenfrage geht; oder aber qualitativ, im Sinne von aussagekräftigen Stellvertretern für einen überschaubaren, tendenziell exklusiven Bestand kontextueller Belege, etwa wenn es um pietistische Bekenntnisschriften aus der Hallenser Gelehrtenschicht geht. Beide Konstellationen weisen jedenfalls die neuhistorische Ausgrenzung extratextueller Kontexte zugunsten einer scheinbar innovativen Intertextualität als gegenstandslos aus. Schließlich bedeutet >Philologieく u. a., dass auch ideelle oder gesellschaftliche Konstellationen wie >Pietismus` oder >Frauenemanzipation nur aus exemplarischen Textzeugnissen rekonstruiert werden können, und dass deren Menge begründet zu limitieren ist. Die inverse Formulierung des gleichen Befundes findet sich bei Neumann und Nünning: Bestimme man den kulturellen Kontext als »Zusammenhang eines Feldes koexistierender Texte und Medien sowie der in ihnen und durch sie konstituierten Diskurse«, dann sei »der intertextuelle Kontext [...] immer auch als der extratextuelle Kontext eines spezifischen literarischen Werks zu verstehen« (Neumann/Nünning 2006, 16).

Zur Sicherung von Relevanz bzw. Wesentlichkeit schließlich, seit jeher die große Krux jeglicher Kontextphilologie, könnten folgende Suchkriterien einen groben Rahmen abgeben: Textsignale erstens in Form von expliziten Bezugnahmen auf den Kontext, zweitens in Form von impliziten Bezugnahmen bzw. Anspielungen auf den Kontext in distinkten Passagen, drittens implizite Bezugnahmen, die sich aus dem Sinnzusammenhang des Textganzen ergeben (vgl. Kramer 2004, 22), viertens formalästhetische Äquivalenzen zwischen Text und textuellem Kontext, fünftens strukturelle Homologien zwischen Text und extratextueller Umgebung, sechstens kulturelles Wissen im Grad der Allgemeinheit oder siebtens solches der Gruppenspezifität für die soziokulturellen Bezugsgruppen des literarischen Artefakts. Um etwa dem Kontext >Weltkrieg` für literarische Texte zwischen 1914 und 1918 Relevanz zuzuschreiben, bedarf es keiner direkten oder indirekten Textsignale, und ebenso wenig, wenn man dem Kontext >Transzendentalphilosophieく Relevanz für die Dichtung der Frühromantik zuschreiben will. 
Zusammengenommen sollen als Grundprämissen also gelten, dass eine fruchtbare kontextualistische Literaturanalyse der Rekonstruktion historischer Semantik bedarf, dass Text und Kontext $\mathrm{zu}$ unterscheiden und $\mathrm{zu}$ hierarchisieren sind, dass unabhängig von der Weite des Gegenstandsbereiches ein form- und gattungsorientierter Literaturbegriff Möglichkeitsbedingung dieser Hierarchisierung ist. Nicht zuletzt liegt unserem Verständnis von Kontextualismus ein Modell literarischer Kommunikation zugrunde, das die drei Komponenten Autor, Leser und Text einschließt - wobei die Akzentsetzung je nach Perspektive oder Fragestellung variieren kann.

\section{Problemgeschichte, Sozialgeschichte, sLiteratur und Wissen Kontexterschließungen}

Der diskutierte Bedarf nach Begrenzungs- und Suchkriterien, nach Einordnungsmöglichkeiten für das je individuelle Vorgehen und schließlich nach einem Nexus zwischen Theorie und Alltagspraxis waren Anlass für eine literaturwissenschaftliche Workshop-Reihe, die wir 2013 unter dem Titel Das Text-KontextProblem: Theoriemodell vs. Forscheralltag? am Institut für Germanistik der Universität Bern veranstaltet haben.

Dabei wurden drei literaturwissenschaftliche Ansätze oder Praxisfelder mit hermeneutischem Hintergrund auf ihre Ergiebigkeit in der literaturgeschichtlichen Praxis und ihr Potential für die regelgeleitete Erschließung von Kontexten ausgeleuchtet: >Literatur und Gesellschaft` bzw. `Sozialgeschichte`, >Literatur und Wissen Sektion sind jeweils einem dieser Ansätze gewidmet und gehen auf die Vorträge von Dirk Werle, Ralf Klausnitzer und Katja Mellmann im Rahmen unseres Workshops zurück. ${ }^{15}$ Bewusst ist nun von Potential bzw. von Möglichkeiten die Rede, denn die drei diskutierten Formate erfüllen nicht durchgehend die Kriterien für >Methode` oder >Theorie` (Köppe/Winko 2007, 285f.). So fallen unter >Sozialgeschichte` bekanntlich verschiedene Theorieangebote, während sich den Formaten >Literatur und Wissen auf einschlägige systematische Diskussionen allenfalls ein proto-methodischer Status zuschreiben lässt - man denke an die ausufernden Definitionsversuche

15 Die Workshops konnten dank der finanziellen Unterstützung der Mittelbauvereinigung der Universität Bern (MVUB) durchgeführt werden. Dafür sei ihr an dieser Stelle herzlich gedankt. 
zur literaturwissenschaftlichen Problemgeschichte (vgl. insbes. die beiden Jahrgänge von Scientia Poetica 2009 und 2010) oder an die Debatten, die sich um den Wissensbegriff und die Modalitäten einer literarischen Wissensgeschichte ranken (vgl. Köppe 2011; Klausnitzer 2008; Borgards/Neumeyer/Pethes/Wübben 2013; Lukoschek 2013). Gleichwohl erlauben es die drei genannten Ansätze, Materialmengen zu ordnen und zu limitieren; d. h. relevante, repräsentative und fruchtbare Text-Kontext-Relationen mit Bezug auf ein klar definiertes Erkenntnisziel C zu modellieren. Schließlich liefern sie in der Summe eine rudimentäre Klassifikationsmöglichkeit für Kontexte, die - wie gesagt - exemplarisch und nicht exhaustiv zu verstehen ist. Allerdings sei an dieser Stelle darauf hingewiesen, dass die Beiträge von Werle, Klausnitzer und Mellmann je eine bestimmte Perspektivierung präsentieren und nicht den Anspruch erheben, den jeweiligen Ansatz zu homogenisieren.

Nun rekurrieren die drei Zugriffsweisen in unterschiedlichem Ausmaße auf vorangehende literaturtheoretische Traditionen, womit sie jeweils ihren besonderen Untersuchungsfokus bzw. -gegenstand erläutern und damit ihren Anspruch auf eigenständige Forschungsfelder erheben. Der Bereich >Literatur und Wissen`, hervorgegangen aus der in den 1970/80er Jahren geführten Debatte um die zwei wissenschaftlichen Kulturen, zählt heute $\mathrm{zu}$ einem für die kulturwissenschaftlich wie die kulturgeschichtlich orientierte Literaturwissenschaft besonders attraktiven Forschungsfeld; im Zentrum des Interesses steht die wechselseitige Relation von Literatur und insbesondere naturwissenschaftlichem bzw. -kundlichem Wissen. Die heutige wie die ursprüngliche, in den 1960/70er aufgekommene sozialgeschichtliche Literaturwissenschaft hingegen hat die verschiedenen Konstellationen und Relationen von Literatur und Gesellschaft im Fokus. Während also diese beiden Forschungsfelder eine Kontexterschließung nach inhaltlichen Kriterien implizieren, erweist sich das >Problem der >Problemkontext $\triangleleft$ der Problemgeschichte als formale Kategorie, die sich damit grundsätzlich von denjenigen der beiden anderen Ansätze unterscheidet. Denn >Problem lässt sich inhaltlich ganz unterschiedlich füllen und kann auch die beiden Bereiche >Wissen ` und >Gesellschaft ` enthalten, insofern literarische Texte auf herausfordernde gesellschaftliche, wissens- oder ideengeschichtliche Konfigurationen mit einem >Problemlösungsangebot` reagieren. Ferner sind die von der Problemgeschichte adressierten Textumgebungen ausschließlich extratextueller Natur, während etwa >Wissen` extra- wie intertextuelle Kontextmengen umfassen kann.

Im Unterschied $\mathrm{zu}$ den beiden anderen Ansätzen impliziert die Problemgeschichte demnach nicht per se einen eingeschränkten Bereich von kontextuellen Umgebungen, mit denen die literarischen Texte in Relation zu setzen sind, sondern verfügt zunächst einmal über einen offenen bzw. deutlich offeneren Kontexthori- 
zont. Das Problem, relevante und repräsentative Kontextbeispiele zu selegieren, stellt sich hier dringender als bei den anderen beiden Zugriffsweisen, wobei Relevanz dem Leitbegriff `Problem` bereits inhärent ist - ebenso wie das Kriterium der Fruchtbarkeit - und insofern von einer sorgfältigen Konturierung des historischen Bezugsproblems abhängt. Die divergierenden Standpunkte in Bezug auf terminologische wie konzeptionelle Definitionen innerhalb der Problemgeschichte vermochten allerdings bislang nicht zu einer befriedigenden Lösung dieser Schwierigkeiten beizutragen. Ungeklärt ist vor allem die Frage nach Definition, Größenordnung und Einheit des zentralen Konzepts >Problem`, für dessen Bestimmung etwa biologisch, strukturalistisch oder auch alltagssprachlich inspirierte Optionen formuliert wurden: >Problem` kann »als Differenz von Sollwert und Istwert« (Eibl 2010, 240) verstanden werden, ähnlich als »Unvereinbarkeit (mindestens) zweier systemrelevanter Größen« (Titzmann 2010, 305) oder aber als »schwierige Aufgabe mit rätselhaftem Charakter, die als innerhalb bestimmter Situationen der srealen Welt` vorkommend wahrgenommen [wird]« (Werle 2009, 258). Während die ersten beiden Definitionen systemisch-überindividuell angelegt sind, ist die dritte deutlich personalisiert: Werles Vorschlag besteht darin, von einem intentionalistischen Problembegriff auszugehen. Der Autor limitiert als regulative Instanz die per se unbegrenzte Menge an Kontexten, wobei ein reduktiver Psychologismus mit der Ausgangsfrage vermieden wird, auf welche realweltlichen Probleme der Autor mit seinem Text reagierte. So konturiert nicht ein individuelles Bewusstsein sondern ein rekonstruierbarer Problemhorizont den Primärkontext eines Textes.

Freilich hat die Problemgeschichte mit diesem sstrukturellen Suchraster nicht den Anspruch, als Methode aufzutreten, sondern versteht sich als Heuristik bzw. »methodologische Maxime« (Werle). Stärker als die beiden anderen Ansätze lenkt sie ihren Fokus auf generische Erklärungsversuche, indem sich ihr Verstehensziel C vielfach als Erläuterungen des Warum von Entwicklungen präsentiert. Die konzeptionelle Zweigliedrigkeit von >Problem` und >Lösung` bzw. in alternativer Fassung von >Frage und >Antwort deutet implizit auf diesen dynamischen oder prozessualen Charakter der Kategorie >Problem $`$ hin, die sich insofern besonders zur Beschreibung von Wandel und Veränderung anbietet. Literarhistorische Entwicklungen können dadurch leicht mit außerliterarischen Konstellationen korreliert werden, wobei allerdings Letztere Erstere zu determinieren scheinen. Diese Kausalitätsproblematik scheint das schwierige Erbe der traditionellen Ideengeschichte zu sein, mit der die literaturwissenschaftliche Problemgeschichte noch verbunden ist.

Als Vorteil erweist sich hingegen die Möglichkeit, dass im Abstraktum >Problem inhaltliche Aspekte und literarische Form zusammengedacht werden können. Problemgeschichte ist mehr als Ideengeschichte im engeren Sinn, verspricht als Basis der Kontextbildung größere Fruchtbarkeit, denn sie umfasst auch die spezifische Sprachbezogenheit des Literarischen, wie sich etwa an der oben 
bereits erwähnten Studie von Ajouri ablesen lässt. Die korrelative Lektüre von philosophischen, naturwissenschaftlichen und fiktionalen Texten soll nicht nur den ideengeschichtlichen Problemkontext erhellen, sondern vielmehr formalästhetische bzw. narratologische Phänomene perspektivieren helfen.

Die in jüngster Zeit vorgenommenen Versuche, das Forschungsfeld von >Literatur und Wissen s systematisch zu erschließen, haben vor allem das Bewusstsein dafür geweckt, dass auch hier ein Konsens kaum erreichbar ist. Stattdessen sind die beiden Bereiche >Literatur « und `Wissen` je nach individuellem Forschungsvorhaben zu konzeptionieren, somit können auch die Text-Kontext-Relationen nur anhand des je konkreten Einzelfalls reflektiert und beschrieben werden. Zunächst ist nach der genauen Bestimmung des Objektbereichs >Wissen $>\mathrm{zu}$ fragen und zu klären, ob man Wissen als mentale Bestände eines Individuums oder eines Kollektivs versteht oder lediglich in seinen repräsentationalen Formen als Text, Bild, Graphik, Formel, Statistik etc. Wer sich für die Korrelationen zwischen Literatur und den protopoetischen oder elementarliterarischen Darstellungsformen des Wissens interessiert, dürfte sich vorwiegend auf intertextuelle bzw. material fixierte Wissenskontexte beziehen, da Gattungsmuster, Erzählschemata, Motive oder Tropenensembles auch Wissensordnungen strukturieren. Hier kann die ästhetischformale Seite des Symbolsystems Literatur ganz konkret in die kontextualistische Literaturanalyse mit einbezogen werden: Es ist je im Einzelfall $\mathrm{zu}$ entscheiden, inwieweit der intertextuelle Wissenskontext für den literarischen Text nicht nur aus historisch-inhaltlichen Gründen, sondern auch aufgrund struktureller Ähnlichkeiten relevant ist.

Vor allem ist auch nach dem >Träger Wissen des Autors, bestimmter Gruppen oder der ganzen Kultur? Der Abstraktionsgrad des zugrundegelegten Wissensbegriff und die damit verbundene Frage der Zuschreibbarkeit - personenbezogenes oder transsubjektives Wissen - regulieren auch die Selektion von Wissenskontexten. Wer Wissen als propositional und personalisiert denkt, als wahre oder wahrheitsfähige gerechtfertigte Meinung, die jemand hat, der wird sich bei der Wahl der Wissenskontexte am Autor orientieren. Wer Wissen hingegen auf einer abstrakteren und systemischen Ebene als transsubjektiven Bestand von Auffassungen konzipiert, die für ein Kollektiv Weltdeutungs-, Sinnstiftungs- $u$.ä. Funktionen haben, der dürfte breitere und vielfältigere Text-Umgebungen berücksichtigen, die nicht an der Person des Textproduzenten angelehnt sind. Damit verbunden wäre dann auch das Problem der Repräsentativität, das eingehender zu reflektieren wäre. Diesen Aufgabenstellungen begegnet Ralf Klausnitzer in seinem Beitrag mit einer praxeologischen Perspektive: Die Reflexion auf die "grundlegenden Praktiken des Beobachtens« (Klausnitzer), die auch für die Literaturwissenschaft relevant sind, soll dazu beitragen, Text-Kontext-Beziehungen zu spezifizieren. 
Für den Bereich von >Literatur und Gesellschaft ‘ haben sich bis heute zwei grundsätzliche Modellierungen durchgesetzt. Nach systemtheoretischem Zuschnitt erscheint die Literatur als Kommunikation, gemäß Pierre Bourdieus Feldtheorie wird sie als Handeln begriffen; beide Varianten nehmen somit eine System- und Funktionsperspektive ein, die sich auf die übergeordneten Strukturen `Gesellschaft bzw. >literarisches Feld « bezieht. Mit einem solchen, nicht primär textorientierten Literaturbegriff droht freilich das Literarische an sich aus dem Blick zu geraten, das Symbolsystem Literatur durch die Fokussierung auf das Sozialsystem Literatur an Bedeutung zu verlieren (vgl. Ort 1992, 419; 2002, 102). In dieser Problematik gründet das vielfach von Kritikern der Sozialgeschichte angeführte Argument, diese arbeite im Grunde lediglich (zu) reduktive Kausalbeziehungen zwischen Text und realweltlichem Kontext heraus und verstehe die Literatur somit ausschließlich als Widerspiegelung gesellschaftlicher Zustände; besonders dringlich stellt sich hier die Frage nach Relevanz und Fruchtbarkeit von Kontexten für eine gattungs- und formbewusste Arbeit am literarischen Text. Katja Mellmann identifiziert in ihrem Beitrag diese reduktive Sichtweise als Erblast der marxistischen Literaturwissenschaft und entwirft ihrerseits ein systemtheoretisches Modell einer Sozialgeschichte der Literatur, das mit einem vierfachen Kontext-Begriff arbeitet und nicht nur auf die Gesellschaft an sich beschränkt ist, sondern auch die »Umwelten von >Gesellschaft««, d. h. also auch »psychische, biologische und physikalische >Kontexte« (Mellmann) umfassen kann. Die auch von Mellmann vorgenommene funktionale Perspektivierung literarischer Kommunikation bietet die Möglichkeit, Texte in ihre je historische Produktions- und Rezeptionssituation einzubetten, die Textumgebung auch aus Leserperspektive $\mathrm{zu}$ bestimmen. Ein solches Vorgehen erlaubt es ebenfalls, literaturgeschichtlichen Wandel zu beschreiben. Als Gelenkstelle der Kontextselektion und -limitation fungieren dabei die »Anschlusskommunikationen« (Mellmann), die der literarische Text evoziert und insofern ein abstrahierendes Konzept, das oberhalb der Inhaltsebene der gesellschaftlichen Phänomene angesiedelt ist und Formfragen mitdenken lässt.

Zusammenfassend scheint in einer Situation des methodischen Pluralismus und der fundamentalen Abwesenheit von Konsens, der noch nicht einmal zu Fragen der Hierarchisierung und Separierung von Texten und Kontexten gefunden werden kann, die größtmögliche Evidenz für das je einzelne Vorgehen ein sinnvolles Desiderat; vielleicht das einzig realisierbare. Unter den Prämissen des hier skizzierten >distinkten Kontextualismus` erlauben es die drei vorgestellten Ansätze, extra- und intertextuelle Kontexte, orientiert am Autor, am Leser oder am überindividuellen kulturellen Wissen, kriteriengeleitet zu selegieren, zu limitieren und dafür Begründungen anzugeben. Den jeweiligen Problempunkten dieser Ansätze gehen die folgenden Beiträge insofern nach, als sie innerhalb der 
Formate >Problemgeschichte`, `Sozialgeschichte bestimmte Perspektivierungen auswählen und Auswege aus Aporien aufzeigen.

Anmerkung: Abstract translated by Alastair Matthews.

\section{Literatur}

Ajouri, Philip, Erzählen nach Darwin. Die Krise der Teleologie im literarischen Realismus: Friedrich Theodor Vischer und Gottfried Keller, Berlin/New York 2007.

Barner, Wilfried, Kommt der Literaturwissenschaft ihr Gegenstand abhanden? Vorüberlegungen zu einer Diskussion, Jahrbuch der Deutschen Schillergesellschaft 41 (1997), 1-8.

Baßler, Moritz, Die kulturpoetische Funktion und das Archiv. Eine literaturwissenschaftliche TextKontext-Theorie, Tübingen 2005.

-, Analyse von Text- und Kontext-Beziehungen, in: Thomas Anz (Hg.), Handbuch Literaturwissenschaft. Gegenstände - Konzepte - Institutionen, Bd. 2: Methoden und Theorien, Stuttgart/ Weimar 2007, 225-233.

Bevir, Mark, The role of contexts in understanding and explanation, in: Hans Erich Bödeker (Hg.), Begriffsgeschichte, Diskursgeschichte, Metapherngeschichte, Göttingen 2002, 159-208.

Borgards, Roland/Harald Neumeyer/Nicolas Pethes/Yvonne Wübben (Hg.), Literatur und Wissen. Ein interdisziplinäres Handbuch, Stuttgart 2013.

Danneberg, Lutz, Kontext, in: Harald Fricke et al. (Hg.), Reallexikon der deutschen Literaturwissenschaft, Bd. 2, Berlin/New York 2000, 333-337.

Eibl, Karl, »Alles Leben ist Problemlösen« - nach 40 Jahren, Scientia Poetica 14 (2010), 238-252.

Erll, Astrid/Simone Roggendorf, Kulturgeschichtliche Narratologie: Die Historisierung und Kontextualisierung kultureller Narrative, in: Ansgar Nünning/Vera Nünning (Hg.), Neue Ansätze in der Erzähltheorie, Trier 2002, 73-115.

Encke, Julia, Augenblicke der Gefahr und der Krieg der Sinne [1914-1934], München 2006.

Fohrmann, Jürgen, Textzugänge. Über Text und Kontext, Scientia Poetica 1 (1997), 207-223.

Gallagher, Catherine/Stephen Greenblatt, Practicing New Historicism, Chicago/London 2000.

Greenblatt, Stephen, Shakespearean Negotiations. The Circulation of Social Energy in Renaissance England, Oxford 1988.

-, The Swerve. How the World Became Modern, New York 2011.

Hahn, Marcus, Gottfried Benn und das Wissen der Moderne, Göttingen 2011.

Heitmann, Annegret, Einführung. Verhandlungen mit dem `New Historicism`, in: Jürg Glauser/ A.H. (Hg.), Verhandlungen mit dem New Historicism. Das Text-Kontext-Problem in der Literaturwissenschaft, Würzburg 1999, 9-23.

Herwig, Malte, Bildungsbürger auf Abwegen. Naturwissenschaften im Werk Thomas Manns, Frankfurt a. M. 2004.

Jahraus, Oliver, Text, Kontext, Kultur. Zu einer zentralen Tendenz in den Entwicklungen der Literaturtheorie von 1980-2000, Journal of Literary Theory 1:1 (2007), 19-44.

Kafitz, Dieter, Décadence in Deutschland. Studien zu einem versunkenen Diskurs der 90er Jahre des 19. Jahrhunderts, Heidelberg 2004.

Klausnitzer, Ralf, Literatur und Wissen. Zugänge - Modelle - Analysen, Berlin 2008.

-, Literaturwissenschaft. Begriffe - Verfahren - Arbeitstechniken [2004], Berlin/Boston 2012.

Köppe, Tilmann (Hg.), Literatur und Wissen. Theoretisch-methodische Zugänge, Berlin 2011. 
-/Simone Winko, Theorien und Methoden der Literaturwissenschaft. Begriffsklärungen, in: Thomas Anz (Hg.), Handbuch Literaturwissenschaft. Gegenstände - Konzepte - Institutionen, Bd. 2: Methoden und Theorien, Stuttgart/Weimar 2007, 285-289.

-/Simone Winko/Fotis Jannidis, Kontextorientierte Theorien und Methoden, in: Thomas Anz (Hg.), Handbuch Literaturwissenschaft. Gegenstände - Konzepte - Institutionen, Bd. 2: Methoden und Theorien, Stuttgart/Weimar 2007, 336-369.

Kramer, Sven, Die Folter in der Literatur. Ihre Darstellung in der deutschsprachigen Erzählprosa von 1740 bis >nach Auschwitzı, München 2004.

Krämer, Olav, Intention, Korrelation, Zirkulation. Zu verschiedenen Konzeptionen der Beziehung zwischen Literatur, Wissenschaft und Wissen, in: Tilmann Köppe (Hg.), Literatur und Wissen. Theoretisch-methodische Zugänge, Berlin 2011, 77-115.

Lauer, Gerhard, Historizität und Interessantheit. Anmerkungen zum Innovationsanspruch der Literaturwissenschaft als Kulturwissenschaft, in: Hartmut Kugler et al. (Hg.), www.germanistik2001.de. Vorträge des Erlanger Germanistentags, Bielefeld 2002, 925-944.

Lukoschek, Katharina, Zur Kodifizierung der Wissenspoetologie (Rezension von: Roland Borgards/ Harald Neumeyer/Nicolas Pethes/Yvonne Wübben (Hg.), Literatur und Wissen. Ein interdisziplinäres Handbuch, Stuttgart/Weimar 2013.), JLT online (13.12.2013), http://nbn-resolving. de/urn:nbn:de:0222-002612 (11.04.2014).

Martin, Dieter, Barock um 1800. Bearbeitung und Aneignung deutscher Literatur des 17. Jahrhunderts von 1770 bis 1830, Frankfurt a. M. 2000.

Montrose, Louis, The Elizabethan Subject and the Spenserian Text, in P. Parker/D. Quint (Hg.), Literary Theory/Renaissance Texts, Baltimore 1986, 303-340.

-, Spenser's Domestic Domain: Poetry, Property, and the Early Modern Subject, in: M. de Grazia et al. (Hg.), Subject and Object in Renaissance Culture, Cambridge 1996, 83-130.

Müller, Jan-Dirk, Einleitung, in: J.-D.M./Elisabeth Müller-Luckner (Hg.), Text und Kontext: Fallstudien und theoretische Begründungen einer kulturwissenschaftlich angeleiteten Mediävistik, München 2007, VII-XII.

Neumann, Birgit/Ansgar Nünning, Kulturelles Wissen und Intertextualität: Grundbegriffe und Forschungsansätze zur Kontextualisierung von Literatur, in: Marion Gymnich/B.N./A.N. (Hg.), Kulturelles Wissen und Intertextualität. Theoriekonzeptionen und Fallstudien zur Kontextualisierung von Literatur, Trier 2006, 3-29.

Nünning, Ansgar, Towards a Cultural and Historical Narratology: A Survey of Diachronic Approaches, Concepts, and Research Projekts, in: Bernhard Reitz/Sigrid Rieuwerts (Hg.), Anglistentag 1999 Mainz. Proceedings, Trier 2000, 345-373.

Ort, Claus-Michael, Vom Text zum Wissen. Die literarische Konstruktion sozio-kulturellen Wissens als Gegenstand einer nicht-reduktiven Sozialgeschichte der Literatur, in: Lutz Danneberg/Friedrich Vollhardt (Hg.), Vom Umgang mit Literatur und Literaturgeschichte. Positionen und Perspektiven nach der »Theoriedebatte«, Stuttgart 1992, 409-442.

-, Sozialgeschichte der Literatur und die Probleme textbezogener Literatursoziologie - anläßlich von Kafkas ıDas Urteilı, in: Oliver Jahraus/Stefan Neuhaus (Hg.), Kafkas ,Urteil und die Literaturtheorie, Stuttgart 2002, 101-125.

Oschmann, Dirk, Die Sprachlichkeit der Literatur, in: Jan Urbich/Alexander Löck (Hg.), Der Begriff der Literatur, Berlin/New York 2010, 409-426.

Panagl, Oswald/Ruth Wodak (Hg.), Text und Kontext: Theoriemodelle und methodische Verfahren im transdisziplinären Vergleich, Würzburg 2004. 
Pross, Caroline, Literarische Archäologie. Perspektiven der Forschung zur >Décadence in Deutschland (Rezension von: Dieter Kafitz, Décadence in Deutschland. Studien zu einem versunkenen Diskurs der 90er Jahre des 19. Jahrhunderts, Heidelberg 2004), IASL online (19.05.2005), http://www.iaslonline.Imu.de/index.php?vorgang_id=1285 (11.04.2014)

Specht, Benjamin, Physik als Kunst. Die Poetisierung der Elektrizität um 1800, Berlin/New York 2010.

Stiening, Gideon, ১Glücklicher Positivismusı? Michel Foucaults Beitrag zur Begründung der Kulturwissenschaften, germanistik.ch. Verlag für Literatur- und Kulturwissenschaft (Oktober 2009), www.germanistik.ch/publikation.php?id=Glücklicher_Positivismus (11.04.2014).

Stöckmann, Ingo, Der Wille zum Willen. Der Naturalismus und die Gründung der literarischen Moderne 1880-1900, Berlin 2009.

Stramm, August, Gedichte, Dramen, Prosa, Briefe, hg. von Jörg Drews, Stuttgart 1997.

Thomé, Horst, Autonomes Ich und Inneres Ausland. Studien über Realismus, Tiefenpsychologie und Psychiatrie in deutschen Erzähltexten (1848-1914), Tübingen 1993.

Titzmann, Michael, Kulturelles Wissen - Diskurs - Denksystem: Zu einigen Grundbegriffen der Literaturgeschichtsschreibung, Zeitschrift für französische Sprache und Literatur 99:1 (1989), 47-61.

-, , Problem und Problemlösung` als literarhistorisches und denkgeschichtliches Interpretationsinstrument, Scientia Poetica 14 (2010), 298-332.

Vogl, Josef, Für eine Poetologie des Wissens, in: Karl Richter/Jörg Schönert/Michael Titzmann (Hg.), Die Literatur und die Wissenschaften 1770-1930. Festschrift zum 75. Geburtstag von Walter Müller-Seidel, Stuttgart 1997, 107-127.

Vollhardt, Friedrich, Kulturwissenschaft. Wiederholte Orientierungsversuche, in: Kathrin Stegbauer/Herfried Vögel/Michael Waltenbauer (Hg.), Kulturwissenschaftliche Frühneuzeitforschung. Beiträge zur Identität der Germanistik, Berlin 2004, 29-48.

Werle, Dirk, Frage und Antwort, Problem und Lösung. Zweigliedrige Rekonstruktionskonzepte literaturwissenschaftlicher Ideenhistoriographie, Scientia Poetica 13 (2009), 255-304.

White, Hayden, Metahistory. The Historical Imagination in Nineteenth Century Europe, Baltimore/London 1973.

Winko, Simone/Fotis Jannidis/Gerhard Lauer, Geschichte und Emphase. Zur Theorie und Praxis des erweiterten Literaturbegriffs, in: Jürn Gottschalk/Tilmann Köppe (Hg.), Was ist Literatur? Basistexte Literaturtheorie, Paderborn 2006, 122-154. 